\title{
Abrupt changes in visual stimulation enhance processing of form and location information
}

\author{
CAROL L. KRUMHANSL \\ Cornell University, Ithaca, New York
}

\begin{abstract}
Two experiments investigated the role that abrupt changes in stimulation play in the processing of visual information. In both experiments, the prestimulus field contained a linear array of alternating characters and the target field contained a single target character in one of the positions of the prestimulus array. The target character was either identical to the character in the prestimulus array in that position (no-form change) or was a different character (form change). In the first experiment, the duration of the prestimulus array was 500 msec, and judgments about both the target's form and location were found to be more accurate in the formchange condition. In the second experiment, the duration of the prestimulus array was varied from 10 to 320 msec. A general decrease was found in performance (in both form and location judgments) as the duration of the prestimulus array was increased. The detrimental effect of increasing the duration of the prestimulus array was larger for no-form-change than for formchange trials. These results are accounted for by a quantitative model that assumes that a stim. ulus onset initiates a brief period of rapid information processing, followed by a period with reduced rate of processing.
\end{abstract}

The experiments to be reported here investigated the effect of rapid changes in stimulation on the processing of figural and position information about visually presented forms. The majority of experimental studies in the literature directed at characterizing the properties of early visual processing have employed experimental designs in which the target form or forms are presented against a previously blank background. That is, target presentations are accompanied by a rapid temporal change in the visual stimulation. This work has yielded descriptions of the nature of visual information and its rate of encoding and decay under these conditions. However, naturally occurring visual stimulation does not for the most part contain abrupt changes. The physically present stimulus does not generally undergo sudden alterations, and relatively little is known about visual information processing in the absence of such stimulus changes. Implicit in most previous work is the assumption that the experimental situation is analogous to retinal stimulation when eye movements occur. There is, however, little direct evidence to support this assumption. The present experiments investigate possible differences in the nature and rate of information extraction in stimulus

This research was supported in part by an award from the Milton Fund to the author and in part by a grant from the National Science Foundation (BNS 76-09959) to W. K. Estes. The helpful comments of W. K. Estes, G. Wolford, and an anonymous reviewer, and the technical assistance of $D$. H. Allmeyer are gratefully acknowledged. Reprint requests should be sent to: Carol L. Krumhansl, Department of Psychology, Uris Hall, Cornell University, Ithaca, New York 14853. sequences in which there is or is not a rapid change accompanying the onset of the target character.

Todd and Van Gelder (1979) investigated the effect of abrupt stimulus changes on visual information processing by comparing performance on trials of two types: "onset" and "no onset." On onset trials, the prestimulus field was blank except for the fixation mark, and the stimulus field contained a single target form. Thus, at the start of the target field, the target position changed from being blank to containing a target form. On the no-onset trials, the prestimulus field contained characters in all possible target positions, and the start of the target field was marked by the offset of the characters in all nontarget positions; the target position remained unchanged. In some experiments of the series, subjects were required only to move their eyes to the target position, and the time to initiate the eye movement was the dependent measure. In other experiments, subjects were required to make speeded forcedchoice identity or category (even vs. odd digit) judgments in the absence of eye movements. In all these different conditions, performance on onset trials was better than performance on no-onset trials. The advantage for the onset trials increased with task complexity and with the distance of the target from the fixation point.

Todd and Van Gelder (1979) interpreted their results in terms of a model utilizing the physiological distinction between sustained and transient visual channels. Physiological evidence suggests there may be two quite distinct subsystems within the visual system, one responsive to abrupt stimulus onsets or 
offsets (transient cells) and one responding in a steady-state manner to continuing stimulation (sustained cells). Breitmeyer and Ganz (1975) and Ikeda and Wright (1972) provide summaries of the properties of these subsystems found in cats and monkeys. Briefly, transient cells are characterized as having briefer onset latencies, shorter response durations, and poorer spatial resolution, and as being located primarily in the parafovea and periphery. In contrast, the sustained cells have longer onset latencies, continuing responses throughout stimulation, and relatively good spatial resolution, and they are located primarily in the fovea. Given these different spatial, temporad, and distributional properties, Breitmeyer and Ganz (1976) and Ikeda and Wright (1972) suggested that the physiologically characterized transient channels would be well suited for coding the presence and approximate location of moving or rapidly changing objects outside the fovea, and the sustained channels may carry information about the detailed figural properties of the visual stimulus, particularly if the stimulus is located in the foveal region.

Todd and Van Gelder (1979) suggest that their finding of better performance in the onset condition than in the no-onset condition might be attributable to the greater involvement of transient channels in the onset condition. Specifically, the rapid change in the target position on the onset trials is presumed to activate transient channels. This transient response may then be used to recruit other resources when necessary, such as the information contained in the sustained channels. Alternatively, the response may be based on information contained entirely within the transient channels, even when the task requires form and category information. They cite the work on recognition of dynamic stimuli by Johansson (1973) and Kozlowski and Cutting (1977) in support of this latter interpretation, arguing that the transient system may carry sufficient information even for the more complex tasks. The greater advantage found by Todd and Van Gelder for onset trials over no-onset trials in the periphery was accounted for by the higher ratio of transient to sustained cells in this region.

- However, the design of the Todd and Van Gelder (1979) study allows two alternative explanations. On their onset trials, the prestimulus field was entirely blank, so that the average luminance in the target position was much greater than the average luminance in the nontarget positions (which were blank throughout the trial). In contrast, the difference on no-onset trials between the luminance in the target position averaged over the prestimulus and target fields and the average luminance in the nontarget positions (which always contained characters in the prestimulus field) was relatively small. In other words, because of the visual persistence of the patterns in the nontarget positions on no-onset trials, the target position may be more difficult to distinguish from the nontarget position on these trials. The interaction found between onset/no-onset conditions and retinal position is also consistent with this interpretation. Sakitt (1975, 1976; Sakitt \& Long, 1978) has argued that visual persistence is a consequence of the relatively poor temporal resolution of rod receptors which are concentrated in the periphery. Consequently, visual persistence, producing the difficulty of distinguishing between target and nontarget positions on no-onset trials, would have a more marked effect when the target appeared in peripheral locations.

A second alternative explanation is suggested by the recent series of experiments by DiLollo (1980). He suggests that an abrupt change in visual stimulation initiates a period of rapid information extraction. At the onset of a stimulus, information is rapidly recruited, but after the stimulus has been present for a certain duration, the rate of information encoding drops considerably despite the fact that the stimulus is still physically present. DiLollo (1980) found evidence for this proposal in a series of experiments using varied stimulus and task situations. In some of these experiments, the task required the temporal integration of a pattern displayed in two successive portions. Performance decreased markedly if the duration of the first portion of the display exceeded about $100 \mathrm{msec}$. In a different forwardmasking paradigm, the effectiveness of the mask was found to decline sharply as the duration of the mask was increased. From these results, DiLollo concluded that there is an initial recruitment phase of approximately $100 \mathrm{msec}$ that is more or less independent of the actual duration of the target. Moreover, he argued that visual persistence, which is presumed to be involved in both -kinds of experimental designs, might better be conceptualized as the product of ongoing neural processes than as the contents of a visual store that begins to decay at stimulus offset. Efron (1973) and Haber and Standing (1969) have also reported an inverse relationship between stimulus durations and estimates of visual persistence, consistent with DiLollo's (1980) proposal.

The notion that a stimulus onset induces an interval of rapid information extraction might also account for the findings of Todd and Van Gelder (1979). In particular, the target in the onset conditions of their experiments would presumably initiate a period of encoding at a high rate and result in the rapid growth of information about the target in that condition. This would serve to direct eye movements and allow accurate judgments about the target character to be made. In contrast, in the no-onset condition the target would be present for some duration before the offset of the characters in the nontarget 
positions; in their experiments, this duration exceeded DiLollo's (1980) estimated value of approximately $100 \mathrm{msec}$ for the initial extraction period. Consequently, processing of the target position after the prestimulus field of fset would occur at a reduced rate, affecting both latency and accuracy measures. This, then, would account for the differences found between their onset and no-onset conditions.

The two experiments to be reported here were directed at evaluating these two alternative accounts. The central purpose of the first experiment was to determine whether better performance would be found for sequences containing rapid onsets in the target position even when the prestimulus fields were equated. The second experiment was designed to test a specific prediction of DiLollo's (1980) proposed initial recruitment stage by varying the duration of the prestimulus array.

\section{EXPERIMENT 1}

The design of the first experiment is shown in Figure 1, which illustrates "no-form-change" and "form-change" trials. Unlike the Todd and Van Gelder (1979) study, the prestimulus fields were identical in the two conditions. On trials of both types, the prestimulus field contained 10 characters (alternating $\times s$ and $+s$ ), the order of which was varied randomly from trial to trial. A masking character was included at either end of the display to approximately equate lateral masking effects across the 10 positions. In this experiment, the duration of the prestimulus field was held constant at $500 \mathrm{msec}$. The target, which appeared in one of the 10 positions, was either an $x$ or $a+$. On no-form-change trials, the target character was identical to the character in the same location in the prestimulus field. The target frame onset was simultaneous with the offset of the prestimulus field, so that the character in the target position was effectively continuously present. That is, for no-form-change trials, no rapid change in the target position accompanied target field onset. On the form-change trials, the target differed from the character preceding it in the prestimulus array, producing an abrupt alteration in that position at the start of the target field. In both conditions, the target frame was followed by a masking field and finally a response frame which indicated the 10 possible target positions.

Subjects were required to both identify the target character (identification judgment) and indicate its location. Previous investigations had indicated the partial independence of naming and locating visual stimuli under certain conditions. Krumhansl and Thomas (1976) found that the accuracy of naming a single letter was independent of the accuracy of specifying its absolute location and concluded that, for single targets, the two kinds of information were processed independently. Also, in a letter-report task, Estes, Allmeyer, and Reder (1976) observed that identification, but not location, accuracy improved significantly with stimulus duration. In contrast, studies requiring the naming and the specification of the relative positions of multiple letters found a correlation between accuracy in the two tasks (Krumhansl, 1977; Krumhansl \& Thomas, 1976, 1977). Indeed, the central assumption of the feature perturbation model proposed by Wolford (1975) is that the letteridentification process is strongly dependent on accurate information about the spatial location of letters and their features. Together, these studies suggest that an independent relationship between the two kinds of information may be observed, but primarily in relatively simple stimulus and response situations. In support of this suggestion, a study by Logan (1975) found evidence for independence when the target was easily discriminable from simultaneously presented noise characters, but not when target and noise characters were visually similar.

The partial independence of the processing of form and location information is also suggested by the properties of the sustained and transient channels. As noted earlier, Breitmeyer and Ganz (1976) and Ikeda and Wright (1972) proposed that transient and sustained channels of the visual system might independently subserve the processing of location and figural information, respectively. This view would seem to predict that location accuracy should be higher in the form-change condition of the present experiment than in the no-form-change condition, owing to the abrupt stimulus change in the form-change condition which may activate the transient channel. In contrast, form judgments may be less accurate in the form change condition because of persistence of the character in the prestimulus array in the target position, which is different from the target. Integrationtype masking (Eriksen, 1966; Haber, 1969) would, thus, tend to obscure the target form in the formchange condition. There was some reason to believe, then, that the stimulus sequence conditions employed here might differentially affect the encoding of target form and position.

An alternative pattern of results might be an advantage for both form and position information in the form-change condition. The transient channel response to the target onset in this condition may serve to alert the visual system to the presence and general location of the target. This information may, in turn, improve the utilization of information contained in the sustained (and possibly also in the transient) channel for both form and location judgments. Or, in Neisser's (1967) terms, the transient response may serve to direct focal attention to the appropriate location for further processing of the figural characteristics of the stimulus. Also, DiLollo's (1980) proposal suggests that the target onset may initiate a period of 
rapid information extraction, and, if both position and form information are encoded during this period, then his view would predict that the formchange condition would produce greater accuracy in both tasks.

\section{Method}

Subjects. The eight subjects who participated in this study were young adults who either volunteered their services or were paid at the rate of $\$ 2.75 / \mathrm{h}$. All subjects had served in similar studies but were naive to the purposes of the present experiment. Each subject was tested individually in two separate experimental sessions that lasted a total of approximately $21 / 2 \mathrm{~h}$. All subjects had either normal or corrected-to-normal visual acuity.

Apparatus. A PDP-12 computer (Digital Equipment Corporation) controlled the stimulus display on the CRT screen, a VR-12 point plot display device (Digital Equipment Corporation) with a fast-decaying phosphor (P31). A frontal headrest was used to fix the viewing distance at $70 \mathrm{~cm}$, and subjects viewed the display through a rectangular slit in a black shield. The experimental room was dimly illuminated. Two rows of response buttons interfaced with the computer were used to collect the data.

Stimulus materials. Figure 1 illustrates sample trials from the no-form-change and form-change conditions. In both conditions, the prestimulus array was displayed for $500 \mathrm{msec}$ and was followed immediately by the target frame, which contained a single $x$ or + in one of the 10 central positions. On no-form-change trials, the target was the same as the character in the corresponding position in the prestimulus array; on form-change trials, the target was different from the character in that position. The duration of the target frame was 60,90 , or 120 msec. The poststimulus frame consisted of $123 \times 3$ grid masking characters and was displayed for 500 msec. Finally, the response frame, which was included to indicate the 10 possible target positions, appeared and remained on the screen until the subject had responded.

The array subtended a visual angle of approximately $6.50 \mathrm{deg}$. The $x$ and + characters and the $3 \times 3$ grid were all constructed using nine points of a $5 \times 5$ grid that was approximately $.28 \mathrm{deg}$ in width and .33 deg in height. Intercharacter spaces were also approximately .28 deg; the fixation point was separated from its neighboring characters by .14 deg.

Procedure. The subjects were told that the following sequence of events would occur on each trial: (1) A fixation point would appear, centered on the CRT display screen. (2) After having fixated this point, the subject was to initiate the trial by pressing any of the 10 buttons in the top row on the button box in front of

SAMPLE TRIAL SEQUENCES

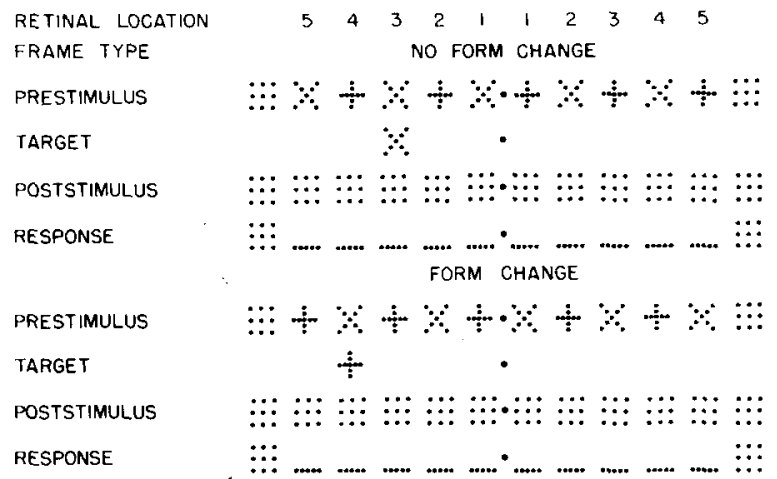

Figure 1. Sample stimalus sequences in the no-form-change and form-change conditions of Expertment 1. (The conditions of Experiment 2 were similar except that the durntion of the preatimulas field was varied and only elght target poitions were used.) him/her. (3) The prestimulus, target, and poststimulus frames, which were described to the subjects, would then be displayed in sequence. (4) The response frame would then appear while the subject made his or her response. The subjects were instructed to indicate which of the 10 locations contained the target character by pressing the corresponding button in the row of 10 buttons. Following this, the subject was to indicate whether the target was an $x$ or $a+$ by pressing one of the two buttons located below the row of 10 buttons. Blocks of trials consisted of four practice trials followed by 120 trials in random order. Each subject was presented with 10 blocks of trials, divided into two experimental sessions.

\section{Results}

The average probability of a correct identification response is shown in the left panel of Figure 2 as a function of the retinal location of the target, the duration of the target, and whether the target was identical to or different from the character contained in the target location in the prestimulus frame. An analysis of variance showed this advantage for the form-change condition over the no-form-change condition to be significant $[F(1,7)=14.27, p<.01]$. In addition, the main effects of target duration and retinal location were significant $[F(2,14)=110.22$ and $F(4,28)=18.87$, respectively, with $p<.001$ in both cases]. These variables also interacted with the formchange/no-form-change variable such that the difference between form-change and no-form-change trials was largest at the shorter target duration and the more peripheral retinal locations. The interaction between form-change/no-form-change condition and target duration gave an $F(2,14)=4.08, p<.05$, and the interaction between form change/no form change and retinal location gave an $F(4,28)=4.30, p<.01$. Finally, accuracy was somewhat higher for targets presented in the right visual field than in the left $[F(1,7)=10,81, p<.05]$, and visual field interacted with retinal location $[F(4,28)=5.62, p<.01]$. An analysis of variance was also performed on the arcsine transformation of the scores. The results of this analysis were, in terms of levels of significance, almost identical to the earlier analysis, with the exception that the interaction between target duration and form change/no form change was no longer significant $[F(2,14)=1.95, p>.15]$.

The average probability of a correct location judgment in each condition is shown in the central panel of Figure 2. As with the identification scores, duration and retinal location had significant effects on performance $[F(2,14)=13.07$, and $F(4,28)=13.68$, $\mathrm{p}<.001$ for both], with location judgments more accurate at longer durations and target positions closer to the fixation point. Furthermore, the interaction of retinal location and duration was significant $[F(8,56)=3.51, p<.01]$, as was the fourthorder interaction $[F(8,56)=2.69, p<.05]$. In terms of location performance, however, the advantage for the form-change trials over the no-form-change trials did not reach statistical significance $[F(1,7)=3.21$, 


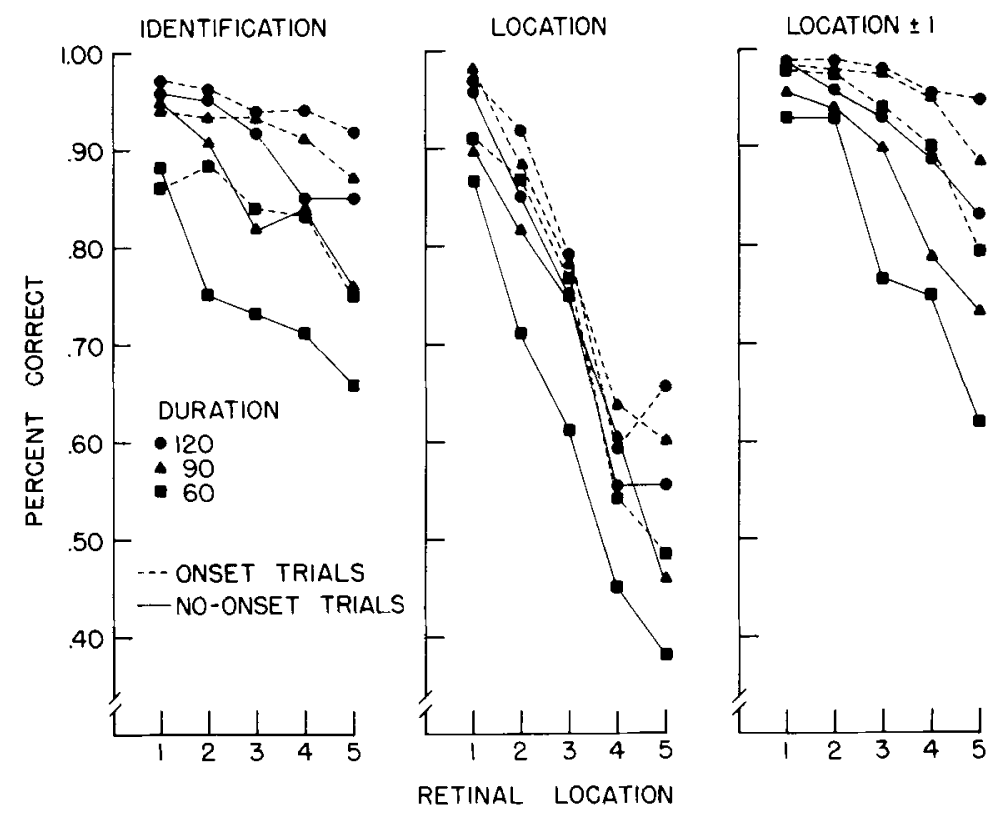

Figure 2. Accuracy of identification (left panel), location (center panel), and location correct within one position (right panel) for Experiment 1 as a function of the position of the target for three different target durations and for form-change and no-form-change conditions.

$\mathrm{p}=.12]$. The analysis of the arcsine transformed data showed the same effects to be significant, except that the onset/no-onset factor now approached significance $[F(1,7)=5.24, p=.056]$, and the interaction between target location and duration was no longer significant $[F(8,56)=1.94, p=.07]$.

Because of the possibility that position judgments might be affected by the presence of the noise character at either end of the display (that is, that subjects might incorrectly assign location responses to the target position because there is an extra character at the ends of the array), a more liberal scoring procedure was used. For the data plotted in the righthand panel of Figure 2, a location response was scored as correct if it was within one position of the correct target position. Using this measure, the advantage for the form-change condition over the noform-change condition was significant $[F(1,7)=6.74$, $\mathrm{p}<.05]$. As with detection performance, the main effects of target duration and retinal location were significant $[F(2,14)=15.70$ and $F(4,28)=10.43$, respectively, with $\mathrm{p}<.001$ in both cases]. These two variables interacted $[F(8,56)=8.19, \mathrm{p}<.001]$ with a larger target duration effect at the more peripheral locations, probably reflecting ceiling effects when the target appeared near the fixation point. Again, both target duration and retinal location interacted with form-change/no-form-change condition. These interactions had values of $F(2,14)=7.24$, and $F(4,28)$ $=4.87$, respectively, with $p<.01$ for both. With the exception of the fourth-order interaction $[F(8,56)$ $=2.38, \mathrm{p}<.05]$, no other variables or interactions were significant. In the analysis of variance on the arcsine transformed data, this fourth-order interaction was nonsignificant, but otherwise the significance levels were similar to those obtained for the untransformed data.

\section{Discussion}

To summarize the results of this first experiment, better performance in both identifying and locating targets was found for form-change trials than for trials containing no form change in the target position. These results extend the findings of Todd and Van Gelder (1979) in three main ways. First, an advantage was found for targets which changed abruptly at the onset of the target field, even though the prestimulus arrays were identical in both conditions. This suggests that the presence or absence of characters in the prestimulus array was not the sole determinant of the differences found in their study. Second, the rapid stimulus changes contained in the stimulus sequences in this and the Todd and Van Gelder study were produced in different ways. In this study, the change was a consequence of altering the form in the target position, whereas in the earlier study the change was produced by presenting the target on a previously blank field. These similarities suggest that both blank-to-target and form-change sequences have analogous effects on the processing of visual information. Finally, the present study found effects of abrupt changes in visual stimulation on accuracy of both naming and locating a target in addition to the previously obtained differ- 
ences in discriminative reaction times and latency of eye movement initiation.

The present experiment does not produce a clear dissociation between the processing of form and location information. At least under these conditions, a form change in the critical location facilitates identity judgments. This is contrary to the simple notion that identification judgments are based on information contained in sustained channels, and that the utilization of this information is equally effective whether or not there is an accompanying transient response. In the form-change condition of the present experiment, the information contained in sustained channels should be relatively poor because of the intrachannel integration of the two different forms contained in the critical position. Apparently, this disadvantage is compensated for in some way by the form change accompanying target onset. The form change may serve as a useful signal to enhance the efficiency of the target-identification process by emphasizing the appropriate subset of form-analyzing channels, sustained channels in the terms of the sustained-transient dichotomy. Alternatively, the response to the stimulus onset may direct focal attentive mechanisms (Neisser, 1967) to the appropriate position, or initiate a period of rapid figural information encoding (DiLollo, 1980). These different accounts will be considered more thoroughly later. It is also possible that the stimulus sequence in the form-change condition contained insufficient changes in low spatial frequency components to activate the transient channels and that a clear dissociation between position and form information would be obtained in a similar stimulus sequence that employed characters with more distinct low spatial frequency content.

It should be noted that the results of this experiment do not necessarily argue for or against the independence of the two types of information, position and form. The results simply indicate that performance in both measures was enhanced by a form change in the stimulus sequence, not that within a particular condition the accuracy of form and location judgments are related. A subsequent analysis of the data from the present experiment did not reveal a consistent correlation on a trial-by-trial basis between the two measures in either the form-change or no-form-change conditions. This is consistent with the single-letter condition of the Krumhansl and Thomas (1976) study. Moreover, this analysis argues against the possibility that the target form judgments are based on a combination of the location information and encoded information about the positions of $X s$ and $+s$ in the prestimulus array. In other words, it might be that subjects have accurate information about the characters in the prestimulus array and the target position, that they know whether or not a form change has occurred, and that they derive from this the identity of the target form. This strategy would produce a correlation, such as that obtained, between the two tasks across conditions. However, it would also predict a trial-by-trial correlation between the two measures within conditions, which was not evident. More direct evidence against the possibility that subjects derive their form judgments from a representation of the prestimulus array is found in the second experiment.

It is also possible that apparent motion produced by the transition between the prestimulus and target fields might have influenced the results in some way. However, there are two reasons that this is unlikely. First, apparent motion was not phenomenologically present in either the form-change or no-form-change conditions. Second, the stimulus onset asynchrony of $500 \mathrm{msec}$ (or the interstimulus interval of $0 \mathrm{msec}$ ) would not be expected to produce strong apparent motion. This possibility was also investigated more directly in the second experiment.

Before turning to that experiment, it should be noted that performance in both form and location tasks improved with target duration and its proximity to the fixation point, as would generally be expected. Each of these factors interacted with the formchange/no-form-change variable. These interactions are of potential interest because of the distributional and temporal differences between transient and sustained channels discussed earlier. Owing to the possibility of ceiling effects in the percent correct measures and the strong dependence of the significance of these interactions on the particular data transformation performed to eliminate possible ceiling effects, these results should be viewed with caution and will not be discussed further here.

\section{EXPERIMENT 2}

The first experiment replicated the basic finding of the Todd and Van Gelder (1979) study showing that an abrupt target onset enhances the processing of information about that target. One possible account of this effect, noted earlier, is in terms of DiLollo's (1980) proposal that the target onset initiates a period of rapid encoding of target information. In the form-change condition, the abrupt change in the target position presumably induces rapid extraction of both form and position information. In contrast, the target in the no-form-change condition has been physically continuous for a duration exceeding the duration of the initial stage of rapid processing. Consequently, little target information is available to the subject during the interval following the offset of the prestimulus array and prior to the poststimulus masking field. (Only information accrued during this interval would serve to discriminate between target and nontarget positions, a discrimination which is required by both location and form judgments.) This, 
then, would explain the main result of the first experiment reported here.

Additional support for the applicability of DiLollo's (1980) proposed initial recruiting stage to the present experimental design might be found if the duration of the prestimulus field were varied. Specifically, this account predicts an interaction between the duration of the prestimulus field and the form-change/noform-change variable. The advantage for formchange over no-form-change trials should be reduced (or even reversed) if the prestimulus field is of brief duration. If the recruitment of the target on no-formchange trials begins with prestimulus field onset, then most of that period of rapid recruitment would fall in the critical interval between prestimulus field offset and postmask onset when the prestimulus field duration was brief. If its duration is longer, the initial recruitment stage would have terminated by the time the critical period began, thus providing little information about the unchanged target. In contrast, the changed target in the form-change condition would presumably initiate the recruitment phase, more or less independently of the duration of the prestimulus field. This, then, would produce an interaction between the two conditions (form-change/no-formchange) and prestimulus array duration. This prediction is tested in the second experiment.

The second experiment, which varied the duration of the prestimulus array, could also be used to evaluate the possible involvement of two other factors discussed earlier. First, it was suggested that subjects might derive their form judgments from information about the characters in the prestimulus array. This would predict a general increase in identity performance as the duration of the prestimulus array was increased, allowing more complete encoding of the prestimulus array. Second, the suggestion was made that apparent motion might be involved in this experimental situation. This would predict that the pattern of results should depend very specifically on the duration of the prestimulus array. Both of these possibilities were evaluated here.

\section{Method}

Subjects. Fifteen adult subjects were each paid $\mathbf{\$ 4 . 5 0}$ for participating in $11 / 2-h$ experimental session.

Stlmulus materials. The stimulus materials were identical to those of Experiment 1 with the following exceptions. First, the target duration was fixed at $90 \mathrm{msec}$ for all trials. Second, the duration of the prestimulus array was varied; the durations used were $10,20,40,80,160$, and 320 msec. Finally, the target might appear in any one of only eight positions, rather than the 10 positions of Experiment 1. Thus, the prestimulus array consisted of eight alternating $x$ and + characters, with a masking character at either end of the array. The intercharacter spaces were adjusted to be approximately $.38 \mathrm{deg}$, so that the array subtended a visual angle of 6.30 deg.

Apparatus and Procedure. Each subject was presented with six blocks of 192 trials in random order. All combinations of experimental variables were presented equally often. The first block of trials was considered practice, and was not included in the data analysis.

\section{Results}

The average probability of a correct identification response is shown in the top panel of Figure 3 as a function of the duration of the prestimulus array, and whether or not there was a form change in the target position at the time of target field onset. An analysis of variance of these results showed a strong detrimental effect of increasing the duration of the prestimulus array $[F(5,70)=15.24, p<.001]$ and a strong interaction of the duration effect with the form-change/no-form-change variable $[F(5,70)$ $=9.08, \mathrm{p}<.001]$. This interaction is seen as the much larger effect of increasing the duration of the prestimulus field for no-form-change trials than for form-change trials. There was, however, no overall effect of form change vs. no form change $[F(1,14)$ $<1]$. As would be expected, performance fell off with the distance of the target from the fixation point $[F(3,42)=55.10, p<.001]$, and there was also an interaction of the retinal location of the target and the duration of the prestimulus field $[F(15,210)=2.20$, $\mathrm{p}<.01]$. This interaction can be described as a larger duration effect at the more peripheral positions. The interaction between form change/no form change and retinal location was not significant. The analysis of the arcsine transformed data yielded similar results.

The bottom panel of Figure 3 shows a similar pattern when the independent measure is the probability of a correct location response. Again, a highly significant effect of prestimulus array duration was found $[F(5,70)=21.74, p<.001]$; this was much larger for trials without a form change in the target location. This interaction between form change/noform change and duration produced an $F(5,70)=$ $13.60, p<.001$. There was a significant overall advantage for form-change trials $[F(1,14)=5.33$, $p<.05]$. A retinal location effect $[F(3,42)=99.68$,

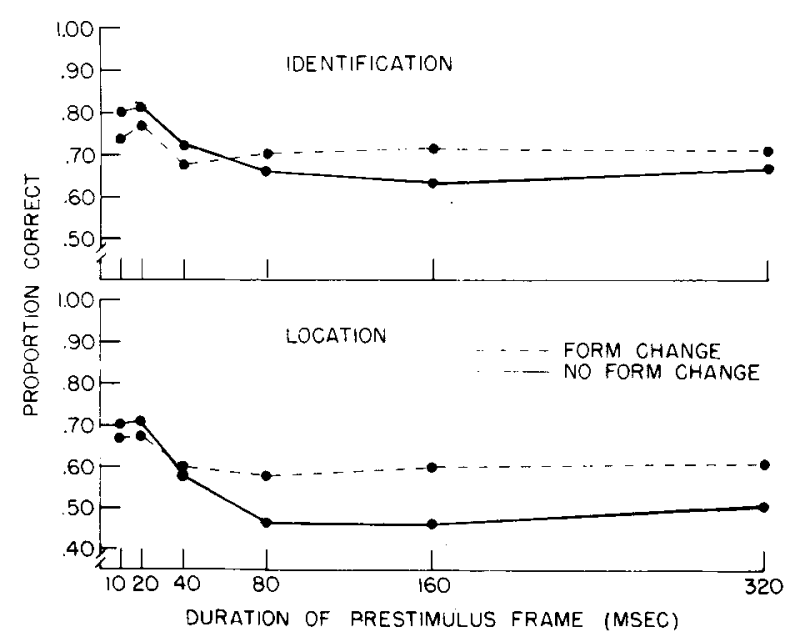

Figare 3. Accuracy of identification and location judgments to Experiment 2 as a function of the duration of the prestimulus array for form-change and no-form-change conditions. 
$\mathrm{p}<.001]$, which interacted with duration $[\mathrm{F}(15,210)$ $=1.73, \mathrm{p}<.05$ ], was found. As with the identification data, a larger effect of increasing the prestimulus array duration was found in the peripheral positions. Here, in addition, there was an interaction between form change/no form change and target location $[F(3,42)=3.03, p<.05]$, which was, however, only marginally significant and not consistently related to target position. Analysis of the arcsine transformed data produced similar results.

\section{Discussion}

The second experiment confirmed the prediction, based on DiLollo's (1980) proposed early recruitment stage, that the duration of the prestimulus array would interact with whether there was or was not a form change in the target position simultaneous with the offset of the prestimulus array (and the onset of the target frame). For the long durations of the prestimulus array, an advantage was again found for the form-change trials. This is consistent with the view that, for the no-form-change targets, the rapid recruitment stage is terminated before the critical interval between the pre- and poststimulus field when the duration of the prestimulus frame is long. However, for the shorter durations of the prestimulus frame, there was a slight advantage for the no-formchange targets over the form-change targets. This reversal may be accounted for if it is assumed that, in the no-form-change condition, persisting information from the target position in the prestimulus array may facilitate judgments about its location and figural properties when the prestimulus array is very brief. In the next section, a quantitative model will be presented that accounts for this interaction with a few simple assumptions.

In addition, a general decrease in the accuracy of both form and location judgments was found as the duration of the prestimulus array increased. That is, performance was lower overall for longer exposures of the prestimulus array. This effect may be understood if one considers that the difficulty of this task is in distinguishing between the target and nontarget positions. As the duration of the prestimulus array increases, there may be a greater probability that a nontarget position is incorrectly judged as containing the target. The difference in luminance (averaged over the prestimulus and target frames) between the target and nontarget positions decreases as the prestimulus array exposure increases. This effect is also predictable from the quantitative model to be presented. It should be noted that this result is entirely inconsistent with the idea that form judgments are derived from information about the characters in the prestimulus array. This predicts that increasing the duration of that array would result in improved accuracy of form judgments, opposite to the result obtained. Finally, explanations in terms of apparent motion, which may in some way produce the ad- vantage for form-change trials, can be rejected because this effect has been found consistently over a wide range $(80-500 \mathrm{msec})$ of prestimulus array durations. Apparent motion is known to be more sensitive to temporal parameters.

\section{A QUANTITATIVE MODEL}

The results of these experiments may be accounted for by a model that makes the following two general assumptions:

(1) There is an initial stage of rapid processing of visual information for a period of time, $t_{c}$, following stimulus onset. This stage is followed (after time $t_{c}$ ) by a period of information extraction that has a rate $b$ times the rate of encoding during the initial stage.

(2) Encoded information decays at the rate $\mathrm{e}^{-\alpha \mathrm{t}}$, where $t$ is the time since the encoding of that information.

The first assumption is equivalent to DiLollo's (1980) proposed initial recruitment stage, except that here it is assumed that encoding does not terminate after time $t_{c}$, but continues at a reduced rate (if $0<b<1$ ). (DiLollo argues that visual persistence is entirely a function of the initial recruitment stage, an assumption which does not have direct bearing on the present discussion.) The second assumption, that the extracted information decays as a negative exponential function, is frequently made in the literature and is chosen for mathematical tractability.

One additional assumption is needed to apply the general model to the particular stimulus situation employed in these experiments. It is:

(3) The probability of a correct form or location judgment is a linear function of the difference between the information available about the target and nontarget positions at the time of poststimulus field onset.

This assumption specifies the difficulty in this experimental situation as one of distinguishing between target and nontarget positions. In other words, the more information persisting from the nontarget positions, the greater is the chance of confusing a nontarget with the target. It is assumed that the subject waits until the end of the target frame to make this judgment because, as a result of the decay of prestimulus characters, the greatest difference between the visual system response to target and nontarget characters would generally occur at that time.

Let $t_{c}$ be the time at which the rapid recruitment period ends after stimulus onset, $t_{p}$ the duration of the prestimulus field, and $t_{t}$ the duration of the target. The response of the visual system to an unchanged target in the no-form change condition at its termination is proportional to $\mathbf{I}_{\mathbf{N C}}$, where $\mathbf{I}_{\mathrm{NC}}$ is given by:

$$
\int_{0}^{t_{p}+t_{t}} e^{-\alpha\left(t_{1}+t_{p}-t\right)} d t=\frac{1-e^{-\alpha\left(t_{t}+t_{p}\right)}}{\alpha} \text {, if } t_{p}+t_{t}<t_{c} \text {, }
$$




$$
\begin{aligned}
& \int_{0}^{t_{c}} e^{-\alpha\left(t_{t}+t_{p}-t\right)} d t+\int_{t_{c}}^{t_{1}+t_{p}} b e^{-\alpha\left(t_{t}+t_{p}-t\right)} d t \\
&=\frac{1}{\alpha}\left[e^{-\alpha\left(t_{t}+t_{p}-t_{c}\right)}\left(1-e^{-\alpha t_{c}}\right)+b\left(1-e^{-\alpha\left(t_{t}+t_{p}-t_{c}\right)}\right)\right], \\
& \text { if } t_{p}+t_{t}>t_{c} \cdot
\end{aligned}
$$

The visual system response to a form-change target at its termination is proportional to $\mathrm{I}_{\mathrm{CH}}$, where $\mathrm{I}_{\mathrm{CH}}$ is given by:

$$
\begin{gathered}
\int_{0}^{t_{t}} e^{-\alpha\left(t_{t}-t\right)} d t=\frac{1-e^{-\alpha t_{t}}}{\alpha}, \text { if } t_{t}<t_{c}, \\
\int_{0}^{t_{c}} e^{-\alpha\left(t_{t}-t\right)} d t+\int_{t_{c}}^{t_{t}} b e^{-\alpha\left(t_{t}-t\right)} d t \\
=\frac{1}{\alpha}\left[e^{-\alpha\left(t_{t}-t_{c}\right)}\left(1-e^{-\alpha t_{c}}\right)+b\left(1-e^{-\alpha\left(t_{t}-t_{c}\right)}\right)\right], \text { if } t_{t}>t_{c} .
\end{gathered}
$$

Finally, the response to a nontarget character in the prestimulus array at the termination of the target frame is proportional to $I_{N T}$, where $I_{N T}$ is given by:

$$
\begin{array}{r}
\int_{0}^{t_{p}} e^{-\alpha\left(t_{t}+t_{p}-t\right)} d t=e^{-\alpha t_{t}} \frac{\left(1-e^{-\alpha t_{p}}\right)}{\alpha}, \text { if } t_{p}<t_{c}, \\
\int_{0}^{t_{c}} e^{-\alpha\left(t_{t}+t_{p}-t\right)} d t+\int_{t_{c}}^{t_{p}} b e^{-\alpha\left(t_{t}+t_{p}-t\right)} d t \\
=\frac{e^{-\alpha t_{t}}}{\alpha}\left[e^{-\alpha\left(t_{p}-t_{c}\right)}\left(1-e^{-\alpha t_{c}}\right)+b\left(1-e^{-\alpha\left(t_{p}-t_{c}\right)}\right)\right], \\
\text { if } t_{p}>t_{c} . \quad \text { (3) }
\end{array}
$$

The probability of a correct response, then, is assumed to be proportional to: $I_{N C}-I_{N T}$ for no-formchange trials, and $I_{\mathrm{CH}}-I_{\mathrm{NT}}$ for form-change trials.
The model was applied to the results from Experiment 2, and Table 1 contains the predicted and observed values for form-change and no-form-change conditions, the six prestimulus field durations, and the two tasks (form and location judgments). An optimal fit was found for $t_{c}=110 \mathrm{msec}, \alpha=.022$, and $b=.80$. The correlation between the predicted and observed values was .920 for the probability of a correct form judgment and .953 for the probability of a correct location judgment. Thus, a quite satisfactory fit between the observed and predicted values was obtained with only three parameters (plus the two regression coefficients which were found separately for the form and location data).

To illustrate how the model accounts for the obtained results, Figure 4 shows the total information available for no-form-change targets, form-change targets, and prestimulus array characters as a function of time since the onset of the prestimulus array. The values plotted are those obtained using the parameters found to give an optimal fit to the data from the second experiment; the information unit is arbitrary. The graph at the top of Figure 4 shows the functions for a short prestimulus array duration of $10 \mathrm{msec}$. Because the sum of $t_{t}+t_{p}=100 \mathrm{msec}$ is less than $t_{c}=110 \mathrm{msec}$, the information about the noform-change target increases throughout the interval between its onset (at $t=0 \mathrm{msec}$ ) and its offset (at $t=100 \mathrm{msec}$ ). The total information about the unchanging target at the time of its offset is indicated by INC. The information about the target in the form-change condition also increases throughout its exposure. The maximal value of this function, achieved at the time of target offset and indicated by $I_{\mathrm{CH}}$, is less than $\mathrm{I}_{\mathrm{NC}}$ because the onset of the formchange target is delayed by $10 \mathrm{msec}$ (the duration of the prestimulus array) as compared with the noform change target. The differences between the

\begin{tabular}{|c|c|c|c|c|c|c|c|c|}
\hline \multirow{3}{*}{$\begin{array}{l}\text { Prestimulus } \\
\text { Duration }\end{array}$} & \multicolumn{4}{|c|}{ Identification } & \multicolumn{4}{|c|}{ Location } \\
\hline & \multicolumn{2}{|c|}{ Form Change } & \multicolumn{2}{|c|}{ No Form Change } & \multicolumn{2}{|c|}{ Form Change } & \multicolumn{2}{|c|}{ No Form Change } \\
\hline & $\mathrm{O}$ & $\mathrm{P}$ & $\mathrm{O}$ & $\mathbf{P}$ & $\mathrm{O}$ & $\mathbf{P}$ & 0 & $\mathrm{P}$ \\
\hline 10 & .743 & .773 & .804 & .793 & .663 & .674 & .699 & .706 \\
\hline 20 & .775 & .755 & .818 & .792 & .669 & .647 & .702 & .705 \\
\hline 40 & .683 & .730 & .726 & .733 & .588 & .606 & .579 & .612 \\
\hline 80 & .701 & .702 & .665 & .655 & .576 & .562 & .459 & .488 \\
\hline 160 & .714 & .701 & .641 & .653 & .599 & .560 & .458 & .485 \\
\hline 320 & .704 & .704 & .677 & .658 & .605 & .566 & .506 & .492 \\
\hline
\end{tabular}
values $I_{\mathrm{NC}}$ and $\mathrm{I}_{\mathrm{CH}}$ correspond to the greater accuracy of form and position judgments at short durations of the prestimulus array in the no-form-change condition. The information from the prestimulus array characters increases throughout its exposure (from $t=0$ to $t=10 \mathrm{msec}$ ) and then decays as a negative exponential function throughout the target ex-

Table 1

Fit of the Model to Data From Experiment 2

Note-The values of the estimated parameters were $t_{c}=110, \alpha=.022, b=.80 . \quad O=$ observed, $P=$ predicted. 


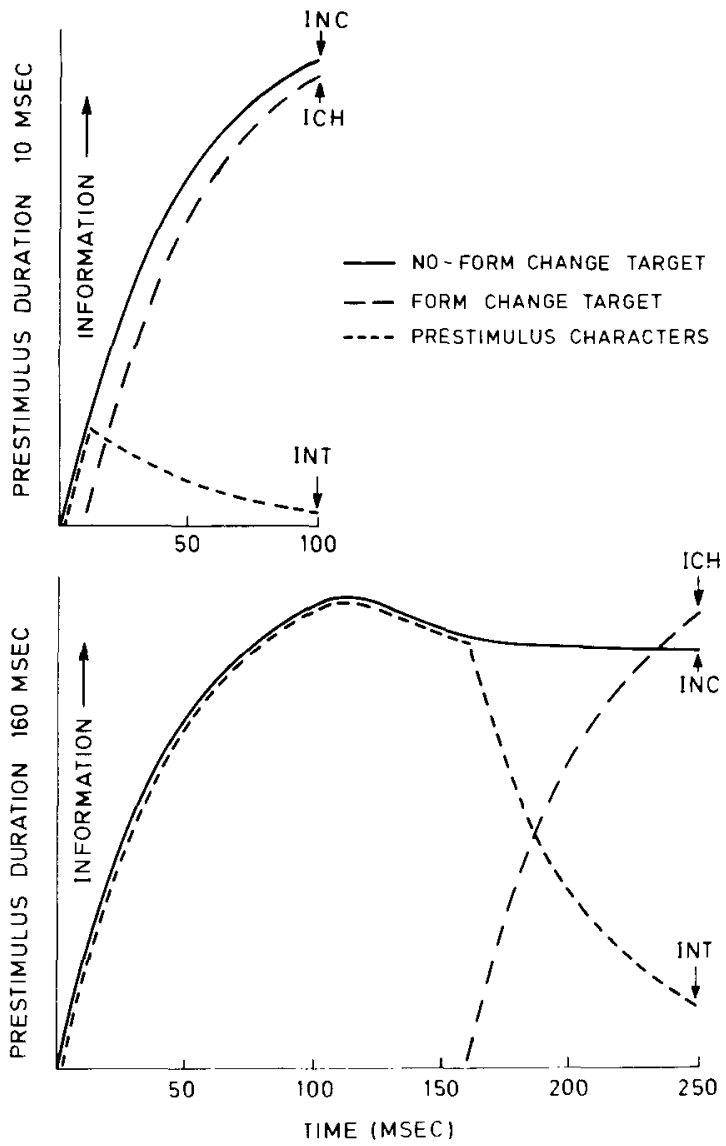

Figure 4. The total information avallable at each point in time after prestimulus array onset as predicted by the model. The theoretical values are plotted for no-form-change targets, form-change targets, and prestimulus army charactern. The values of the functons at time of target offset are denoted $I_{\mathrm{NC}}, I_{\mathrm{CH}}$, and $\mathrm{I}_{\mathrm{NT}}$, respectively. The graphs at the top of the figure correspond to a short prestimulus array duration of 10 msec; the graphs at the bottom of the figure correspond to a longer prestimulus array duration of 160 msec.

posure, reaching a value of $I_{N T}$ at the termination of the target field. It is assumed that performance is directly related to the difference between the target information and the prestimulus array information at the time of target field offset, in this case at $t=$ $100 \mathrm{msec}$. Because relatively little information persists from the nontarget prestimulus array characters at this time, accuracy in both the form-change and the no-form-change conditions is relatively high for short prestimulus array durations.

The lower graphs show the corresponding values for a longer prestimulus array duration of $160 \mathrm{msec}$. Because the sum of $t_{t}+t_{p}=250$ msec exceeds the critical duration, $t_{c}=110 \mathrm{msec}$, the function for noform-change targets first increases to a maximal value at $110 \mathrm{msec}$, and then decreases to a lower asymptotic value. This decrease is due to the lower rate of information extraction from the target position in the no-form-change condition after the critical duration of $110 \mathrm{msec}$. In contrast, the informa- tion from the target position increases throughout its exposure (beginning at $t=160 \mathrm{msec}$ and ending at $t=$ $250 \mathrm{msec}$ ). In this case, the information from the form change target at the time of target offset, $\mathrm{I}_{\mathrm{CH}}$, exceeds that from the no-form-change target, $I_{N C}$. This difference, then, accounts for the greater accuracy found for form-change targets than for noform-change targets for longer durations of the prestimulus array. The information from the prestimulus array characters first increases to the maximum value during the interval from $t=0$ to $t=110 \mathrm{msec}$ and then approaches the lower asymptotic value until the offset of the prestimulus array, at which time the function decays exponentially. Because the information from the prestimulus array characters has achieved a relatively high value during its duration, there is considerable information, $\mathrm{I}_{\mathrm{NT}}$, persisting from nontarget positions at the time of target offset (at $t=250 \mathrm{msec}$ ). This value is greater than that for shorter prestimulus array durations, which accounts for the general decline in performance in both formchange and no-form-change conditions as the duration of the prestimulus array increased. However, because the nontarget prestimulus array characters are subject to the same processes as the target character, the value of $I_{\mathrm{NT}}$ will also approach an asymptotic value. This predicts the relatively constant performance in both form-change and no-form change conditions found in the experiment for the longer durations of the prestimulus array.

The estimated value of $t_{c}=110 \mathrm{msec}$ for the duration of the rapid recruitment stage agrees well with DiLollo's (1980) estimate. The present results indicate that information continues to be extracted subsequent to that initial period; the estimated value of the $b$ parameter was .80 . This means that the rate of encoding following the initial recruitment stage was reduced, although it maintained a fairly high rate. However, because of decay, the total information available at any point in time approaches an asymptotic level proportional to the parameter $b$. A number of studies (e.g., Cohene \& Bechtoldt, 1974, 1975; Townsend, 1981; Townsend, Taylor, \& Brown, 1971) have found asymptotic limits to performance with increasing exposure durations consistent with the model. It should be noted that, because of the particular stimulus sequences employed, the finding of the first experiment that performance increased with target duration is not inconsistent with the model. As the target duration increases, the visual system response to the distractor characters in the nontarget positions of the prestimulus array would decay more by the time of target offset. This corresponds to a decreased value of $I_{\mathrm{NT}}$ at the time of target offset with longer target durations. This decrease in $\mathrm{I}_{\mathrm{NT}}$ would allow for more accurate discriminations between target and nontarget positions, producing better performance for targets of longer duration. Paradigms in 
which increased target durations allow eye movements or enable recoding of information into a form not subject to decay would also be expected to show increased performance with longer target durations.

There was no evidence from fitting the model that longer initial recruitment stages applied to form than to location processing mechanisms. Nor was the estimated reduced rate of encoding during the second stage different in the two cases. That is, the optional values of $t_{c}$ and $b$ were not found to be different for the identity and position data, as would be expected given the similar effects found in these two dependent measures. In this connection, it should be noted that the position judgment required quite precise information (distinguishing the target position from the seven other possible target positions on the trial). This judgment might be based in part on figural properties of the stimulus array as a whole. Consequently, both form and position judgments in this experiment may be derived from information processed by the same mechanisms and, therefore, be subject to the same temporal and rate parameters.

Finally, the model predicts that forward-masking effects should be reduced for longer mask durations, and this is the effect found by DiLollo (1980). When the mask exceeds the estimated value of $t_{c}$, the information persisting from the mask will decline and thus produce less interference with the subsequent target stimulus. In the present design, this would predict that integration-type masking of the target character in the form-change condition should decrease with the duration of the prestimulus array. This may partially account for the improvement of the formchange condition relative to the no-form-change condition for longer prestimulus durations. It does not, however, account entirely for the results, because performance in the form-change condition exceeds that in the no-form-change condition for long prestimulus array durations. Although forwardmasking effects may be reduced, the advantage for form-change trials suggests that additional factors are involved. The present model attributes the difference to changes in the rate of encoding over time.

\section{SUMMARY AND CONCLUSIONS}

The two experiments reported here found that form changes at target field onset improved the accuracy of both identifying and locating the target. This effect was observed for longer durations of the prestimulus field. When the prestimulus array was brief, the opposite difference was obtained. These results were accounted for by a model that assumes that the form change initiated a period of rapid processing of information from the target position, information that was used for both the required form and location judgments. DiLollo (1980) has also proposed an early recruitment stage of visual informa- tion processing and has given a similar estimate of its duration. His proposal was supported by two tasks that were very different from that employed in the present experiments. Thus, there is convergent evidence from quite dissimilar experimental designs for the existence of an early stage of visual processing following stimulus onset or, in the present case, a change in the form contained in a given spatial location.

Neither this nor the earlier series of studies (DiLollo, 1980) found any direct evidence for the differential involvement of the physiologically characterized transient and sustained channels. In the present context, processing of form and position information were not dissociated by either the presence or absence of a rapid change in the stimulus sequence or the duration of the prestimulus array. It is possible that the rather complex nature of the position judgment requires figural information about the stimulus array similar to that required for the identification judgment, or alternatively that the stimulus sequences may have contained insufficient changes in low spatial frequency components to activate transient channels. In other words, the stimulus situation may not have been ideal for producing a position response based solely on the response of transient channels. In addition, the retinal location of the target did not interact consistently or reliably with the presence or absence of an abrupt stimulus change in either of the two tasks. An interaction of this sort is of potential interest because of the distributional characteristics of sustained and transient channels found in physiological investigations. As noted earlier, this interaction may have been masked in part by the general decline in performance found with retinal eccentricity. The initial period of rapid encoding assumed by the model may reflect early transient responding (possibly joined somewhat later by sustained responding), which is then followed by a period of neural processing in only sustained channels. However, the present results do not provide any direct support for the possibility, although they are not inconsistent with it.

Another explanation for the present results based on neural processing mechanisms and entirely consistent with the present model comes from the work of Berman and Stewart $(1978,1979)$. They proposed a mathematical model that assumes that the net level of visual activity at any point in time is moderated by impedance in the visual system, possibly produced by lateral inhibition. This impedance is assumed to build up over time. Consequently, the net activity to a stimulus first increases as a function of time, and then decreases to some asymptotic value. This model was used to account for a number of spatial and temporal effects in visual perception. Most relevant to the present discussion is their application of the model to the temporal Broca-Sulzer effect (Broca \& 
Sulzer, 1902; Corwin, 1978; Corwin \& Giambalvo, 1974; Corwin \& Green, 1978; Mansfield, 1973; Marks, 1974). The Broca-Sulzer effect is the variation found in brightness judgments as a function of the duration of the stimulus. These judgments first increase with stimulus duration and then decrease to some constant level. If the brightness judgments are assumed to be some weighted average of the visual system response over the duration of the stimulus, then the decline in brightness judgments for longer stimulus durations may be accounted for by the decrease in activity after continuing stimulation due to impedance in the system (Berman \& Stewart, 1978, 1979). It should be noted that the time course of the Broca-Sulzer effect is quite comparable with what would be predicted by the model presented in this article. Thus, both the present results and the BrocaSulzer effect (as well as DiLollo's, 1980, findings) may all depend on an early stage of rapid recruitment followed by a stage of reduced processing, possibly due to inhibitory neural connections as suggested by Berman and Stewart (1978, 1979).

An alternative possibility is that the present results reflect attentional factors. According to this view, the stimulus onset or change may direct attentional capacities to the appropriate target position, thereby enhancing the processing of its position and form. At present, there is no way to distinguish this hypothesis from the view that the rate of information extraction is itself affected by the presence or absence of an abruptly changing stimulus component. Either of these accounts would have the outcome that the net amount of information available about the target form depends on whether or not the stimulus sequence provides an effective signal from the appropriate position.

In summary, this investigation suggests that visual information processing of steady-state stimulation has characteristics that are different from those found in the processing of rapidly changing stimulation. The results reported here indicate that the rate of encoding declines after the stimulus has been present continuously for a period exceeding approximately $100 \mathrm{msec}$. The extraction of both position and form information were found here to be affected similarly. The processing of other properties of visual stimuli may or may not follow a similar time course. Differences other than simply rate of encoding may also be found between changing and steady-state stimulation. Characterizations of visual information processing derived from experimental situations with abrupt temporal variations in stimulation may thus not generalize to more naturalistic, predominantly steady-state visual environments.

\section{REFERENCES}

Berman, S. M., \& Stewart, A. L. Laterally induced impedance effects in vision. Journal of Mathematical Psychology, 1978, 18, 73-99.
Berman, S. M., \& Stewart, A. L. A unified model for the combined temporal and spatial Broca-Sulzer effect. Biological Cybernetics, 1979, 34, 171-179.

BreitmeYen, B. G., \& GANZ, L. Implications of sustained and transient channels for theories of visual pattern masking, saccadic suppression, and information processing. Psychological Review, 1976, 83, 1-36.

Broca, A., \& Sulzer, D. La sensation lumineuse en fonction du temps. Journal de Physiologie et de Pathologie Generale, 1902, 4, 632-640.

Cohene, L. S., \& Bechtoldt, H. P. Visual recognition as a function of stimulus offset asynchrony and duration. Perception \& Psychophysics, 1974, 15, 221-226.

Cohene, L. S., \& Bechtoldt, H. P. Visual recognition of dotpattern bigrams: An extension and replication. American Journal of Psychology, 1975, 88, 187-199.

Corwin, T. R. The effect of retinal eccentricity on the BrocaSulzer phenomenon. Vision Research, 1978, 18, 865-867.

Corwin, T. R., \& Giambalvo, V. Effects of simultaneous contrast on temporal brightness enhancement. American Journal of Optometry and Physiological Optics, 1974, 51, 175-181.

Corwin, T. R., \& Green, M. A. The Broca-Sulzer effect in a Ganzfeld. Vision Research, 1978, 18, 1675-1678.

DiLoLLo, V. Temporal integration in visual memory. Journal of Experimental Psychology: General, 1980, 109, 75-97.

Efron, R. An invariant characteristic of perceptual systems in the time domain. In S. Kornblum (Ed.), Attention and performance IV. New York: Academic Press, 1973.

ERIKsen, C. W. Temporal luminance summation effects in backward and forward masking. Perception \& Psychophysics, 1966, 1, 87-92.

Estes, W. K., Allmeyer, D. H., \& Reder, S. M. Serial position functions for letter identification at brief and extended exposure durations. Perception \& Psychophysics, 1976, 19, 1-15.

HABER, R. N. Repetition, visual persistence, visual noise and information processing. In K. N. Leibovic (Ed.), Information processing in the nervous system. New York: Springer, 1969.

Haber, R. N., \& Standing, L. G. Direct measures of shortterm visual storage. Quarterly Journal of Experimental Psychology, 1969, 21, 43-54.

IKEDA, H., \& WRIGHT, M. J. Receptive field organization of "sustained" and "transient" retinal ganglion cells which subserve differential functional roles. Journal of Physiology, 1972, 227, 769-800:

JoHansson, G. Visual perception of biological motion and a model for its analysis. Perception \& Psychophysics, 1973, 14, 201-211.

Kozlowski, L. T., \& CutTing, J. E. Recognizing the sex of a walker from a dynamic point light display. Perception \& Psychophysics, 1977, 21, 575-580.

Krumhansl, C. L. Naming and locating simultaneously and sequentially presented letters. Perception \& Psychophysics, 1977, 22, 293-302.

Krumhansi, C. L., \& Thomas, E. A. C. Extracting identity and location information from briefly presented days. Perception \& Psychophysics, 1976, 20, 243-258.

Krumhansl, C. L., \& Thomas, E. A. C. Effect of level of confusability on reporting letters from briefly presented visual displays. Perception \& Psychophysics, 1977, 21, 269-279.

LoGAN, G. D. On the relation between identifying and locating masked targets in visual search. Quarterly Journal of Experimental Psychology, 1975, 27, 451-457.

Mansfield, R. J. W. Brightness function: Effect of area and duration. Journal of the Optical Society of America, 1973, 63, 913-920.

Marks, L. E. Sensory processes. New York: Academic Press, 1974.

Neisser, U. Cognitive psychology. New York: Appleton-CenturyCrofts, 1967.

SAKITT, B. Locus of short-term visual storage. Science, 1975, 190, 1318-1319.

Sakitt, B. Iconic memory. Psychological Review, 1976, 83, 257-276. 
SakitT, B., \& Long, G. M. Relative rod and cone contributions in iconic storage. Perception \& Psychophysics, 1978, 23, 527-536.

Todd, J. T., \& VAN Gelder, P. Implications of a transientsustained dichotomy for the measurement of human performance. Journal of Experimental Psychology: Human Perception and Performance, 1979, 5, 625-638.

Townsend, J. T. Some characteristics of visual whole report behavior. Acta Psychologica, 1981, 47, 149-173.
Townsend, J. T., Taylor, S. G., \& Brown, D. R. Lateral masking for letters with unlimited viewing time. Perception \& Psychophysics, 1971, 10, 375-378.

Wolfond, G. Perturbation model for letter identification. Psychological Review, 1975, 82, 184-199.

(Manuscript received May 24, 1982;

revision accepted for publication September 3, 1982.) 